\title{
Extended faint source detection in astronomical hyperspectral images
}

\author{
Jean-Baptiste Courbot ${ }^{\mathrm{a}, \mathrm{b}, *}$, Vincent Mazet ${ }^{\mathrm{a}}$, Emmanuel Monfrini $^{\mathrm{c}}$, Christophe Collet $^{\mathrm{a}}$ \\ a ICube, Université de Strasbourg - CNRS, 67412 Illkirch, France \\ ' Univ Lyon, Univ Lyon1, Ens de Lyon, CNRS, CRAL UMR5574, F-69230 Saint-Genis-Laval, France \\ c SAMOVAR, Département CITI, CNRS, 91011 Évry, France
}

\section{A R T I C L E I N F O}

\section{Keywords:}

Hyperspectral images

Hypothesis testing

Generalized likelihood ratio

Spatial enhancement

Multiple observations

Astronomical images

\begin{abstract}
A B S T R A C T
The circum-galactic medium consists in gas orbiting around galaxies, whose faintness prevents any complete and easy detection. A powerful tool to detect such pattern can be found in using hyperspectral imaging. Nevertheless, detection in hyperspectral datacubes faces various problems, including well-fitted signal and noise descriptions to ensure further discrimination. A specificity of astronomical images resides in dealing with faint and very noisy signals. In this paper, we introduce a new constrained generalized likelihood ratio test adapted to the problem and a compound test to exploit most of the available information. We also investigate the use of both spatial information and multiple observations on a single scene, to enhance robustness. Numerical experiments on synthetic data are performed to quantify the gain of the different approaches. Finally, results on real hyperspectral astronomical data are presented, which may map for the first time observation of the circumgalactic medium around faint and distant galaxies.
\end{abstract}

\section{Introduction}

\subsection{Problem formulation}

Galaxies evolve by interaction with their environment through different mechanisms. Their immediate environment, known as the Circum-Galactic Medium (CGM), is the place to study these mechanisms. The surrounding neutral gas can be ionized and then be detectable through the Lyman-alpha emission line. This emission line is generally strong in young star-forming galaxies known as LymanAlpha Emitters (LAE). However, the CGM emission is extended, its morphology is unknown and its brightness is much fainter than the emission of the galaxy itself. Until recently [1] it has escaped direct detection, but with the advent of new hyperspectral imagers, it is now possible to detect the CGM at high redshift, at a time where we expect that most interaction should take place. Making progress towards an unbiased and robust detection of the CGM is then of considerable interest for upcoming astrophysical studies.

We propose in this paper a general unsupervised detection strategy for faint extended sources in Hyperspectral Images (HSI or datacube) based on hypothesis testing. This formulation is widespread and often takes the form of two hypotheses describing the presence/absence of the signal. A test is then set to choose between the two alternatives, by the mean of a test statistic thresholding. In the HSI, the tests are applied on spectra, so that the statistic yields a detection map. One might see the test as a contrast function: an image well contrasted between signal and noise implies that the test statistic is more relevant. Furthermore, astronomical HSI present several peculiarities, including:

- They have a non-negligible degree of sparsity, and spectra mostly consist in emission or absorption lines plus an eventual smooth continuum component. This spectral sparsity is well exploited in the context of restoration [2] and source separation [3].

- Long exposure times are needed when probing faint, distant objects. The total exposure time can sum up to several hours, and generally cannot be obtained at once because of cosmic rays, saturation or night duration. Therefore, the observation is actually an average of several observations. Nevertheless, we also have access to the individual (stacked) observations.

Keeping in mind these specificities, we now describe previous work on detection in HSI.

\subsection{Previous works in HSI detection}

When considering the detection of target spectra in hyperspectral imagery, one can select non-parametric or parametric methods. The non-parametric methods do not rely on the knowledge or estimation of the background distribution and its parameter. Some of these methods

\footnotetext{
* Corresponding author at: ICube, Université de Strasbourg - CNRS, 67412 Illkirch, France.

E-mail address: jb.courbot@unistra.fr (J.-B. Courbot).
} 
are related to order statistics, such as the FDR control [4,5], which are robust to changes in noise level but not to outliers. This makes it delicate to choose in the eventuality of a spatially wide target. Other methods from this family, specifically designed to handle outliers, rely on extreme value theory [6]. This kind of method is useful when the noise distribution is hard to estimate from the observation, and when the target is seldom encountered in the image. In our application, the target is weak, so its impact on the estimation will be negligible, and since it may cover a significant part of the HSI, we do not need to resort to extreme value theory.

Therefore, parametric methods may be better suited to our problem. They can be classified in two categories: the supervised methods, in which the target spectrum is known prior to detection, and the unsupervised methods, in which it is not. In the first category, well-known detectors in HSI are the Adaptive Matched Filter (AMF) [7] and the Adaptive Cosine/Coherence Estimator (ACE) [8]. Both use the input data to estimate the background distribution, but rely on a known spectrum to perform the detection. Hence, they are well-adapted to look for known anomalies in a HSI [9]. Besides, they have the Constant False Alarm Rate (CFAR) property: their false alarm rate does not depend on nuisance parameters [10]. This property is particularly appealing for practical implementations. Other approaches include Orthogonal Subspace Projectors [11] in which background or signal knowledge is exploited to perform projection prior to signal/background separation, and hybrid detectors such as [12-14].

When the target spectra are unknown, it is necessary to resort to unsupervised techniques. The most used as a benchmark in automatic HSI source detection is the Reed-Xiaoli (RX) algorithm [15] and improvements (e.g. [16]). This method measures how anomalous a spectrum is, given a background and its estimated covariance matrix. More recent work in unsupervised detection also includes projection pursuit (PP) $[17,18]$. The aim of such a method is to project data onto a 1D space in order to optimize some criterion, e.g. the outliers-sensitive kurtosis. In [19], the authors also propose a Spatio-Spectral Gaussian Random Field (SS-GRF) modeling for noise. This method also behaves as an anomaly detector, since the signal structure is unconstrained. Other methods from the unsupervised family aim at building a reference from the observation alone. These methods [20-22] use machine learning techniques to learn the signal target under various constraints (e.g. with sparsity). However, when targeting for emission lines in astronomical images, one can assume there exists a general description of the target line (e.g. Gaussian) that can be used as prior within the detection method.

Relatively to the previously cited works, we address the problem of source detection, with partial knowledge of the target to detect. The hypothesis testing framework appears well suited to properly describe what is unknown and what is known. One of the most common hypothesis testing formulation for detection in HSI is the Generalized Likelihood Ratio (GLR) [23]. It uses a re-formulation of NeymanPearson's optimal Likelihood Ratio test [24], replacing the signal and noise knowledge by Maximum Likelihood (ML) estimates. This test does not present direct optimality or CFAR properties, but appears to perform well in numerous cases (see [25] for a study of GLR properties). Improvements were recently made on the GLR formulation, either with sparse constraints [26] to have a better signal characterization or with priors on signal distribution [27].

Among previous studies, a few instances $[19,28]$ consider spatial relationships between spectra. Other work on this topic includes local summation methods [29], Hidden Markov Random Field modeling [30], cluster modeling [31] and marked point processes [32].

\subsection{Article outline}

Throughout this paper, we make use of assumptions related to astronomical HSI analysis. First, the sources spectra present few nonzero coefficients, modeling an emission line. Spatially, their spectra vanish from their center, meaning that a few central spectra are bright whereas the others are faint. Furthermore, the spectra in the bright and faint regions remain similar, because they physically share some amount of gas. To summarize: we seek at locating spectrally-located, spatially-extended, consistent sources in an HSI.

Assumptions concerning the instrument are also necessary. In particular, the instrument Point Spread Function (PSF) should not be neglected with respect to pixel size. Note that the PSF is assumed separable into a spatial Field Spread Function (FSF) and a spectral Line Spread Function (LSF). Moreover, several observations of the same scene, as well as their average, are available. Finally, the noise distribution is assumed multivariate Gaussian in this average, which is correct given the MUSE instrument's behavior (see e.g. [33]).

The main contributions of our work are the following:

- We propose a two-step detection strategy, to perform first a bright source detection and then a faint source detection. The first step addresses the unsupervised dectection of the brighter source. Then, given the resulting bright detection map, the second step performs the detection of similar, fainter spectra in the remaining of the HSI.

- We introduce a new constrained GLR test which considers the similarity with a given spectra. We show that this test has the CFAR property.

- This test leads to a compound test, considering the similarity with respect to a spectra set. This test is used as the second step of the proposed detection strategy. We show that its false alarm rate has an upper bound, which remains independent from noise level.

- The models are also extended to make use of the spatial relationship between spectra, within a local neighborhood.

- The use of multiple observations of a single scene is also investigated to exploit most available information. Experiments show this way does not provide noticeable improvements (Section 3.2).

- Then, we present numerical experiments on the methods with the help of simulated HSI. This allows us to assess method improvements and to compare with others methods [15,19] through Receiver Operating Characteristic (ROC) curve analysis.

- Results on real astronomical data from the MUSE integral field unit [34] are finally presented. They could provide for the first time a statistical mapping of the CGM orbiting around galaxies.

This paper is an extension of prior works reported in [35]. The improvements consist in rephrasing the proposed tests within a constrained GLR framework, exhibiting properties of the tests, considering stacked observations, extending experimental validations, and comparing with an alternative version and with the SS-GRF method from [19].

The paper outline is the following. The proposed models are reported in Section 2. Then, numerical experiments and results are given in Section 3, as well as comparison with state-of-the art method. The application on real MUSE data is finally presented in Section 4, with results on distant, faint galaxies and their environment.

\section{Models}

In this section, we present the models for source detection. As stated in the introduction, the process is split into two steps, detecting first a bright source (Section 2.1) and then its faint counterpart (Section 2.2). Then, in Section 2.3 the models are enriched by considering spatial features and multiple observations. An error analysis is finally presented in Section 2.4, investigating type I error (false alarm rate) analytical properties.

Throughout this paper, we deal with various forms of GLR tests. Most of them will have a constraint, such as the sparsity-enforcing $\mathrm{GLR}_{\mathrm{ls}}^{(\mathrm{ID})}$ proposed in [28]. For brevity we note $\mathcal{T}$ as being any GLR test with catalog constraint (see below). Several test variations are investigated, including or not spatial and observation-wise features, simi- 
Table 1

Detection tests summary. Letters in quote denote the subscripts of the tests and corresponding thresholds. " $\checkmark$ ", $\chi$ ", “-” label wheter the corresponding models are used, not used or not applicable.

\begin{tabular}{|c|c|c|c|c|c|c|}
\hline & Name & Section & Neighborhood & Observations & Compound & Similarity \\
\hline & & & "n" & "o" & "c" & "s" \\
\hline \multirow[t]{3}{*}{ Bright } & $\mathcal{T}$ & 2.2 & $x$ & $x$ & - & - \\
\hline & $\mathcal{T}_{\mathrm{n}}$ & 2.3 .1 & $\checkmark$ & $x$ & - & - \\
\hline & $\mathcal{T}_{\text {no }}$ & 2.3 .2 & $\checkmark$ & $\checkmark$ & - & - \\
\hline \multirow[t]{4}{*}{ Faint } & $\mathcal{T}_{\mathrm{s}}^{\hat{\mathbf{x}}_{b}}$ & 2.2 .1 & $x$ & $x$ & $x$ & $\checkmark$ \\
\hline & $\mathcal{T}_{\mathrm{cs}}^{\mathcal{B}}$ & 2.2 .2 & $x$ & $x$ & $\checkmark$ & $\checkmark$ \\
\hline & $\mathcal{T}_{\mathrm{ncs}}^{\mathcal{B}}$ & 2.3 .1 & $\checkmark$ & $x$ & $\checkmark$ & $\checkmark$ \\
\hline & $\mathcal{T}_{\text {nocs }}^{\mathcal{B}}$ & 2.3 .2 & $\checkmark$ & $\checkmark$ & $\checkmark$ & $\checkmark$ \\
\hline
\end{tabular}

larity constraint and compound testing. Table 1 summarizes the test variations, names and corresponding paper section. Each test requires a threshold, which will be denoted $\xi$, with the subscript corresponding to the test name.

We consider an HSI containing the source of interest, denoted $\left(\mathbf{y}_{S}\right)_{s \in \mathcal{S}}, \mathcal{S}$ being the HSI spectra set. For brevity, the distribution of the random variable $\mathbf{Y}_{s}$, whose realization is $\mathbf{y}_{s}$, is noted $p\left(\mathbf{y}_{s}\right)$; and likewise for the other variables of interest. The spectral hypothesis allows us to use a catalog $\mathbf{C}$, containing $J$ columns $\mathbf{c}_{j}$. In practice, they will be Gaussian lines, representing potential Lyman-alpha lines convolved by the LSF. In this paper, $\mathcal{B}$ denotes the bright spectra set, and we have $s \in \mathcal{S}$ for any spectra, $b \in \mathcal{B}$ for the detected bright spectra (in the first step), $f \in \mathcal{F}=\mathcal{S} \backslash \mathcal{B}$ for the remaining spectra (proceed at the second step). Finally, estimations are denoted with a " $\wedge$ " over the variable of interest.

\subsection{Bright source detection}

This step aims at detecting the bright source. The hypotheses to test are the following $\forall s \in \mathcal{S}$ :

$\mid \begin{aligned} & \mathcal{H}_{0}: \mathbf{y}_{s}=\boldsymbol{\epsilon}_{s} \\ & \mathcal{H}_{1}: \mathbf{y}_{s}=\mathbf{x}_{s}+\boldsymbol{\epsilon}_{s}\end{aligned}$

The additive noise $\boldsymbol{\epsilon}$ on the spectral axis is a realization of a random variable following a multivariate zero-mean normal distribution of covariance matrix $\boldsymbol{\Sigma}$, noted $\mathcal{N}(\mathbf{0}, \boldsymbol{\Sigma})$. We assume that the spectral content $\mathbf{x}_{s}$ of the bright source can be described with a line $\mathbf{c}_{j}$ from $\mathbf{C}$ and a multiplicative factor $\alpha_{s}$.

As $\mathbf{x}_{s}$ is unknown, it is necessary to resort to a GLR test. We use the constrained GLR test from [28], which yields:

$\mathcal{T}\left(\mathbf{y}_{s}\right)=\frac{\max _{\mathbf{c}_{j}, \alpha_{s}} p\left(\mathbf{y}_{s} \mid \mathbf{c}_{j}, \alpha_{s}, \mathcal{H}_{1}\right)}{p\left(\mathbf{y}_{s} \mid \mathcal{H}_{0}\right)} \underset{\mathcal{H}_{0}}{\stackrel{\mathcal{H}_{1}}{\gtrless} \xi .}$

Under the multivariate normal noise hypothesis, $\mathcal{T}$ reduces in:

$$
\begin{aligned}
\mathcal{T}\left(\mathbf{y}_{s}\right) & =\max _{j, \alpha_{s}} \exp \left(-\frac{\alpha_{s}^{2}}{2} \mathbf{c}_{j}^{\top} \boldsymbol{\Sigma}^{-1} \mathbf{c}_{j}+\alpha_{s} \mathbf{c}_{j}^{\top} \boldsymbol{\Sigma}^{-1} \mathbf{y}_{s}\right) \\
& =\max _{j} \exp \left(-\frac{\hat{\alpha}_{s, j}^{2}}{2} \mathbf{c}_{j}^{\top} \boldsymbol{\Sigma}^{-1} \mathbf{c}_{j}+\widehat{\alpha}_{s} \mathbf{c}_{j}^{\top} \boldsymbol{\Sigma}^{-1} \mathbf{y}_{s}\right)
\end{aligned}
$$

where $\widehat{\alpha}_{s}$ is the ML estimate of $\alpha_{s}$ :

$\widehat{\alpha}_{s}=\arg \max _{\alpha_{s}} p\left(\mathbf{y}_{s} \mid \alpha_{s}, \mathbf{c}_{j}, \mathcal{H}_{1}\right)=\frac{\mathbf{c}_{j}^{\top} \boldsymbol{\Sigma}^{-1} \mathbf{y}_{s}}{\mathbf{c}_{j}^{\top} \boldsymbol{\Sigma}^{-1} \mathbf{c}_{j}}$.

Then, the test expression from Eq. (2) reduces to: $\frac{\left(\mathbf{c}_{\hat{j}}^{\top} \Sigma^{-1} \mathbf{y}_{s}\right)^{2}}{\mathbf{c}_{\hat{j}}^{\top} \Sigma^{-1} \mathbf{c}_{\hat{j}}} \underset{\mathcal{H}_{0}}{\gtrless} 2 \ln (\xi) ;$

where:

$\widehat{j}=\arg \max _{j} p\left(\mathbf{y}_{s} \mid \widehat{\alpha}_{s}, \mathbf{c}_{j}, \mathcal{H}_{1}\right)$.

Furthermore, the analytical form (5) makes it possible to express the false alarm rate thanks to the chi-square distribution with one degree of freedom.

This test has been shown to be more efficient than the classical GLR in the case of sparse signals [28]. It will be extended to consider richer information (spatial features, stacked observations) in Section 2.3. The test from Eq. (2) is applied on all $s \in \mathcal{S}$ and the $\mathcal{H}_{1}$ decision region forms the spectra set $\mathcal{B}$. This set will be used as a reference for the extended faint source detection step in the next section.

\subsection{Extended faint source detection}

From now on, we consider the set $\mathcal{B}$ fixed. This step aims at detecting the extended faint source in the remaining data $\mathcal{F}=\mathcal{S} \backslash \mathcal{B}$. The faint spectra are assumed to be similar to the bright ones. First, a new constrained GLR test is introduced in Section 2.2.1, accounting for the similarity between bright and faint spectra. Then, a compound test is proposed in Section 2.2.2, so that most of the available information is exploited.

\subsubsection{Similarity constrained GLR Test}

Let $\mathbf{y}_{b}$ and $\mathbf{y}_{f}$ be two observed spectra, respectively bright $(b \in \mathcal{B})$ and faint $(f \in \mathcal{F})$. The model assumes, under $\mathcal{H}_{1}$, that the fixed $\mathbf{x}_{b}$ and the unknown $\mathbf{x}_{f}$ are described by one catalog line, up to a multiplicative factor $\beta_{f, b} \in \mathbb{R}^{+}$. The two hypotheses for testing a faint $\mathbf{y}_{f}$ are:

$\mathcal{H}_{0}: \mathbf{y}_{f}=\boldsymbol{\epsilon}_{f} ;$

$\mathcal{H}_{1}: \mathbf{y}_{f}=\mathbf{x}_{f}+\boldsymbol{\epsilon}_{f}=\beta_{f, b} \mathbf{x}_{b}+\boldsymbol{\epsilon}_{f}=\beta_{f, b} \alpha_{b} \mathbf{c}_{j}+\boldsymbol{\epsilon}_{f} ;$

where $\boldsymbol{\epsilon}_{f}$ is a realization of zero-mean multivariate normal noise distribution, with covariance matrix $\boldsymbol{\Sigma}$. Let $\widehat{\mathbf{x}}_{b}=\widehat{\alpha}_{b} \mathbf{c}_{\hat{j}}$ be the fixed ML estimation of $\mathbf{x}_{b}$, where $\widehat{\alpha}_{b}$ and $\hat{j}$ are computed with Eqs. (4) and (6) respectively.

Definition 2.1. Given the reference $\widehat{\mathbf{x}}_{b}$, the GLR test with similarity constraint, $\mathcal{T}_{\mathrm{s}}^{\hat{\mathbf{x}}_{b}}$, is defined as:

$$
\begin{aligned}
\mathcal{T}_{\mathrm{s}}^{\hat{\mathbf{x}}_{b}\left(\mathbf{y}_{f}\right)}= & \frac{\max _{\mathbf{x}_{f}} p\left(\mathbf{y}_{f} \mid \mathbf{x}_{f}, \mathcal{H}_{1}\right)}{p\left(\mathbf{y}_{f} \mid \mathcal{H}_{0}\right)} \\
& =\frac{\max _{f, b} p\left(\mathbf{y}_{f} \mid \beta_{f, b} \widehat{\mathbf{x}}_{b}, \mathcal{H}_{1}\right)}{p\left(\mathbf{y}_{f} \mid \mathcal{H}_{0}\right)} \\
& =\frac{p\left(\mathbf{y}_{f} \mid \widehat{\beta}_{f, b} \widehat{\mathbf{x}}_{b}, \mathcal{H}_{1}\right)}{p\left(\mathbf{y}_{f} \mid \mathcal{H}_{0}\right)} ;
\end{aligned}
$$

where $\widehat{\beta}_{f, b}$, is the ML estimate of $\beta_{f, b}$.

The ML estimate of $\beta_{f, b}$ yields:

$\widehat{\beta}_{f, b}=\arg \max _{\beta_{f, b}} p\left(\mathbf{y}_{f} \mid \beta_{f, b}, \widehat{\mathbf{x}}_{b}, \mathcal{H}_{1}\right)=\frac{\widehat{\mathbf{x}}_{b}^{\top} \boldsymbol{\Sigma}^{-1} \mathbf{y}_{f}}{\widehat{\mathbf{x}}_{b}^{\top} \mathbf{\Sigma}^{-1} \widehat{\mathbf{x}}_{b}}$.

Then, $\mathcal{T}_{\mathrm{s}}^{\hat{\mathbf{x}}_{b}}$ can be rewritten with the ML estimate from Eq. (9). Denoting $\widehat{\mathbf{x}}_{f}=\widehat{\beta}_{f, b} \widehat{\mathbf{x}}_{b}$, the logarithm of $\mathcal{T}_{\mathrm{s}}^{\widehat{\mathbf{x}}_{b}}$ is written as: 


$$
\begin{aligned}
\ln \left[\mathcal{T}_{\mathrm{s}}^{\hat{\mathbf{x}}_{b}}\left(\mathbf{y}_{f}\right)\right] & =\widehat{\mathbf{x}}_{f}^{\top} \boldsymbol{\Sigma}^{-1} \mathbf{y}_{f}-\frac{1}{2} \widehat{\mathbf{x}}_{f}^{\top} \boldsymbol{\Sigma}^{-1} \widehat{\mathbf{x}}_{f} \\
& =\widehat{\beta}_{f, b} \widehat{\mathbf{x}}_{b}^{\top} \boldsymbol{\Sigma}^{-1} \mathbf{y}_{f}-\frac{\widehat{\beta}_{f, b}^{2}}{2} \widehat{\mathbf{x}}_{b}^{\top} \boldsymbol{\Sigma}^{-1} \widehat{\mathbf{x}}_{b} \\
& =\frac{1}{2} \frac{\left(\widehat{\mathbf{x}}_{b}^{\top} \boldsymbol{\Sigma}^{-1} \mathbf{y}_{f}\right)^{2}}{\widehat{\mathbf{x}}_{b}^{\top} \boldsymbol{\Sigma}^{-1} \widehat{\mathbf{x}}_{b}} .
\end{aligned}
$$

Therefore, the test results in:

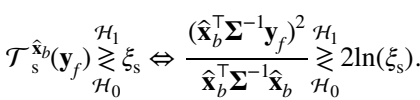

This expression allows us to obtain $P_{\mathrm{FA}}\left(\mathcal{T}_{\mathrm{s}}^{\hat{\mathrm{x}}_{b}}\right)$ in Section 2.4; proving that this test has the CFAR property.

Let us mention that this model and the associated test were inspired by the "LR-MP $\beta$ " test from [26]. The difference are:

1. only the first step of the Matching Pursuit from the LR-MP $\beta$ is used. This avoids setting an input target correlation threshold;

2. we test any pair $(b, f)$ of pixels, not only the contiguous ones;

3. we do not draw the same conclusions as [26] on the $P_{\mathrm{FA}}$ test statistic (see Section 2.4).

\subsubsection{Compound similarity constrained GLR test}

So far, the proposed test addresses a spectrum $\mathbf{y}_{f}, f \in \mathcal{F}$ given a fixed spectrum $\mathbf{y}_{b}$. Given the set $\mathcal{B}$, one can use for $\mathbf{y}_{b}$ a spectrum from the sample, or its averaged spectrum $1 /|\mathcal{B}| \sum_{b \in \mathcal{B}} \mathbf{y}_{b}$. However, using this average under-uses the available information and may lower its statistical variety. We propose therefore to use this test given each $\mathbf{y}_{b}$, i.e. using the whole set $\left(\mathbf{y}_{b}\right)_{b \in \mathcal{B}}$. To preserve an additive analytical formulation (based on the test logarithm, see Eq. (11)), we use the product of each individual component.

Definition 2.2. Given a reference set $\mathcal{B}$ and the corresponding $\left(\widehat{\mathbf{x}}_{b}\right)_{b \in \mathcal{B}}$, the compound similarity constrained GLR test, $\mathcal{T}_{\mathrm{cs}}^{\mathcal{B}}$, is defined as:

$\mathcal{T}_{\mathrm{cs}}^{\mathcal{B}}\left(\mathbf{y}_{f}\right)=\prod_{b \in \mathcal{B}} \mathcal{T}_{\mathrm{s}}^{\hat{\mathbf{x}}_{b}}\left(\mathbf{y}_{f}\right)$.

With Eq. (11), the analytical formulation is:

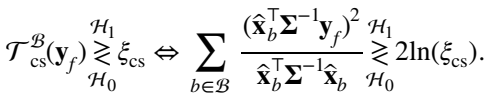

This analytical expression also allows us to search for $P_{\mathrm{FA}}\left(\mathcal{T}_{\mathrm{cs}}^{\mathcal{B}}\right)$. We will show in Section 2.4 that an upper bound can indeed be found on this value.

Let us remark that when performing individual tests $\mathcal{T}_{\mathrm{s}}^{\hat{\mathbf{x}}_{b}}\left(\mathbf{y}_{f}\right)$ in the computation of $\mathcal{T}_{\mathrm{cs}}^{\mathcal{B}}\left(\mathbf{y}_{f}\right)$, the estimates $\hat{\mathbf{x}}_{b}$ may differ (in shape, position or intensity) between locations $b$. This ensure all component of an eventually complex bright source are accounted for.

\subsection{Enriching the models}

This section is dedicated to two enrichments of the model presented so far. First, we consider using spatial features (Section 2.3.1), and then we discuss the use of multiple observations stacking (Section 2.3.2).

\subsubsection{Spatial features}

The previous tests only consider the spectral dimension, whereas the spatial dimension is ignored. We see in this section that considering the spatial FSF is interesting. Indeed, at low Signal to Noise Ratio (SNR), the signal of interest may be lost in a single spectrum, while it does still have a significant presence if its local neighborhood is considered. This spreading is best described by the FSF, which may vary according to the considering wavelength [36]. We assume that the FSF can be described by wavelength-dependent parameters, and spatially fades quickly enough to be windowed within a few pixels. Let us remark that in the case of MUSE observation, the FSF parameter are always available (see e.g. [36]).

To take the FSF into account, the data is transformed so that each site $s \in \mathcal{S}$ contains jointly the original spectrum and its neighbor's spectra within a limited local neighborhood $\mathcal{V}_{s}$, as a stacking. The catalog is replicated in a similar way with FSF weights, yielding:

$\forall s \in \mathcal{S}, \mathbf{y}_{s}^{\mathrm{N}}=\left(\mathbf{y}_{v}\right)_{v \in s \cup V_{s}} ; \mathbf{c}_{j}^{\mathrm{N}}=\mathbf{F}_{j}, 1 \leq j \leq J ;$

where $\mathbf{F}$ is the matrix representing the FSF. Noting $f=\left[f_{1}, \ldots, f_{K}\right]^{\top}$ the $K$ FSF coefficients, it is written as:

$\mathbf{F}=\left[f_{1} \mathbf{I}_{\Lambda}, \ldots, f_{K} \mathbf{I}_{\Lambda}\right]^{\top}=f \otimes \mathbf{I}_{\Lambda} ;$

where $\mathbf{I}_{\Lambda}$ is the $\Lambda$-dimensional identity matrix, and $\otimes$ stands for the Kronecker product.

The tests will involve the estimation of the noise covariance matrix $\boldsymbol{\Sigma}$, which now contains spatio-spectral terms linking an observed spectrum $\mathbf{y}_{s}$ to its neighbors within $\mathcal{V}_{s}$. We assume here and in the following that the noise is not spatially correlated, meaning that the estimation of $\boldsymbol{\Sigma}$ can be constrained to be block-diagonal:

$$
\boldsymbol{\Sigma}=\left[\begin{array}{ccccc}
\boldsymbol{\Sigma}_{1} & \mathbf{0}_{\Lambda} & \cdots & \cdots & \mathbf{0}_{\Lambda} \\
\mathbf{0}_{\Lambda} & \ddots & \ddots & \ddots & \vdots \\
\vdots & \ddots & \ddots & \ddots & \vdots \\
\vdots & & \ddots & \ddots & \mathbf{0}_{\Lambda} \\
\mathbf{0}_{\Lambda} & \cdots & \cdots & \mathbf{0}_{\Lambda} & \boldsymbol{\Sigma}_{K}
\end{array}\right]
$$

where $\boldsymbol{\Sigma}_{n}$ is the spectral covariance matrix of the $n$-th spectrum in the spectra local neighborhood, and $\mathbf{0}_{\Lambda}$ is the $\Lambda \times \Lambda$ zero matrix.

In consequence, we define a bright source detection test using spatial features, noted $\mathcal{T}_{\mathrm{n}}$, with the test from Eq. (2), the spectra from Eq. (14) and the covariance matrix from Eq. (16):

$\mathcal{T}_{\mathrm{n}}\left(\mathbf{y}_{f}\right)=\mathcal{T}\left(\mathbf{y}_{f}^{\mathrm{N}}\right) \underset{\mathcal{H}_{0}}{\stackrel{\mathcal{H}_{1}}{\gtrless} \xi_{\mathrm{n}}}$

Note that the $\mathrm{GLR}_{1 \mathrm{~s}}^{(3 \mathrm{D})}$ from [28] is a particular case of this test in the white noise framework, i.e. with $\boldsymbol{\Sigma}=\mathbf{I}_{K \Lambda}$.

Similarly, we rephrase the compound similarity GLR test, $\mathcal{T}_{\mathrm{cs}}^{\mathcal{B}}$ from Eq. (12):

$\mathcal{T}_{\text {ncs }}^{\mathcal{B}}\left(\mathbf{y}_{f}\right)=\mathcal{T}_{\text {cs }}^{\mathcal{B}}\left(\mathbf{y}_{f}^{\mathrm{N}}\right) \underset{\mathcal{H}_{0}}{\stackrel{\mathcal{H}_{1}}{\gtrless}} \xi_{\text {ncs }}$.

Note that this procedure is not equivalent to deconvolve before testing data. Indeed, such a two-step procedure makes the statistical properties assessment difficult, because of the induced dependence (see however [5]). Instead, the convolution is considered within the test.

\subsubsection{Multiple observations}

As stated in the introduction, astronomical imaging needs long exposure times, resulting in multiple observations of the same field because of sensor saturation effect. Whereas optimal fusion methods have been developed [37], using averaged observations as in the previous sections is generally sufficient. However, information may be lost in the averaging process. Hence, we propose here to extend the models toward multiple, stacked observations. Note that we do not expect the signal of interest to change from one observation to another, and that registration errors are considered negligible, which is the case with well-calibrated ground instruments in astronomy. We consider a simple model, in which the FSF is the same for all observations.

The principle is somewhat simpler than for the spatial feature, as observations are not weighted (unlike local neighboring pixels). Stacking observations from the observation set $O$ yields:

$\forall s \in \mathcal{S}, \mathbf{y}_{s}^{\mathrm{NO}}=\left(\mathbf{y}_{o, s}^{\mathrm{N}}\right)_{o \in O} ; \mathbf{c}_{j}^{\mathrm{NO}}=\left(\mathbf{c}_{j}^{\mathrm{N}}\right)_{o \in O}, 1 \leq j \leq J$. 
Again, the estimation of a covariance matrix on the data from Eq. (19) implies considering observation-related correlations. Whereas the signal is expected to be consistent over multiple observations, this is not the case for noise. Therefore we assume there is no interobservation correlation and constrain the $\boldsymbol{\Sigma}$ estimation to be blockdiagonal. This result, as in Eq. (16), in the following covariance matrix:

$$
\boldsymbol{\Xi}=\left[\begin{array}{cccccc}
\boldsymbol{\Sigma}^{(o=1)} & \mathbf{0}_{K \Lambda} & \ldots & \ldots & \mathbf{0}_{K \Lambda} \\
\mathbf{0}_{K \Lambda} & \ddots & \ddots & \ddots & & \vdots \\
\vdots & \ddots & \ddots & \ddots & \vdots \\
\vdots & & \ddots & \ddots & \mathbf{0}_{K \Lambda} \\
\mathbf{0}_{K \Lambda} & \cdots & \cdots & \mathbf{0}_{K \Lambda} & \boldsymbol{\Sigma}^{(o=|O|)}
\end{array}\right]
$$

where $\boldsymbol{\Sigma}^{(o=n)}$ is the covariance matrix including spatial and spectral terms from Eq. (16), taken for the $n$-th observation, and $\mathbf{0}_{K \Lambda}$ is the $K \Lambda \times K \Lambda$ zero matrix.

We can extend the formulation for the constrained GLR with spatial feature $\mathcal{T}_{\mathrm{n}}$ (from Eq. (17) towards stacked observations. Using the spectra from Eq. (19) and the covariance matrix from Eq. (20):

$\mathcal{T}_{\text {no }}\left(\mathbf{y}_{s}\right)=\mathcal{T}\left(\mathbf{y}_{s}^{\mathrm{NO}}\right) \underset{\mathcal{H}_{0}}{\stackrel{\mathcal{H}_{1}}{\gtrless}} \xi_{\text {no }} ;$

and following the same principle, the extended formulation for the compound similarity GLR test and spatial features, $\mathcal{T}_{\text {ncs }}^{\mathcal{B}}$, yields:

$\mathcal{T}_{\text {nocs }}^{\mathcal{B}}\left(\mathbf{y}_{s}\right)=\mathcal{T}_{\text {cs }}^{\mathcal{B}}\left(\mathbf{y}_{s}^{\mathrm{NO}}\right) \underset{\mathcal{H}_{0}}{\stackrel{\mathcal{H}_{1}}{\gtrless} \xi_{\text {nocs }}}$.

Note that there is, to our knowledge, no existing model accounting for multiple observations in hyperspectral detection. This aspect seems indeed specific to the astronomical framework.

\subsection{Error budget}

It is generally appealing, but not always feasible, to express the type I error $\left(P_{\mathrm{FA}}\right)$ as a function of the test thresholds. In this section we give two properties concerning the $P_{\mathrm{FA}}$ of the tests $\mathcal{T}_{\mathrm{s}}^{\hat{\mathrm{x}}_{b}}$ and $\mathcal{T}_{\mathrm{cs}}^{\mathcal{B}}$.

Proposition 2.1. Given the reference $\widehat{\mathbf{x}}_{b}$, the GLR test with similarity constraint, noted $\mathcal{T}_{\mathrm{s}}^{\hat{\mathrm{x}}_{b}}$, has the CFAR property: the false alarm rate only depends of the test threshold.

Proof. A reformulation of Eq. (11) yields:

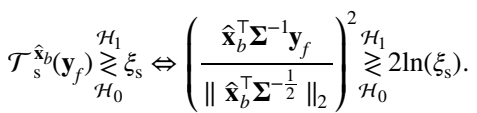

Furthermore:

$$
\begin{aligned}
\mathbf{y}_{f} \stackrel{\mathcal{H}_{0}}{\sim} \mathcal{N}(\mathbf{0}, \mathbf{\Sigma}) & \Rightarrow \widehat{\mathbf{x}}_{b}^{\top} \boldsymbol{\Sigma}^{-1} \mathbf{y}_{f} \stackrel{\mathcal{H}_{0}}{\sim} \mathcal{N}\left(0,\left\|\widehat{\mathbf{x}}_{b}^{\top} \boldsymbol{\Sigma}^{-\frac{1}{2}}\right\|_{2}^{2}\right) \\
& \Rightarrow \frac{\widehat{\mathbf{x}}_{b}^{\top} \boldsymbol{\Sigma}^{-1} \mathbf{y}_{f}}{\left\|\hat{\mathbf{x}}_{b}^{\top} \boldsymbol{\Sigma}^{-\frac{1}{2}}\right\|_{2}} \stackrel{\mathcal{H}_{0}}{\sim} \mathcal{N}(0,1) \\
& \Rightarrow 2 \ln \left[\mathcal{T}_{\mathrm{s}}^{\hat{\mathbf{x}}_{b}}\left(\mathbf{y}_{f}\right)\right] \stackrel{\mathcal{H}_{0}}{\sim} \chi_{1}^{2} ;
\end{aligned}
$$

where $\chi_{1}^{2}$ denotes a chi-squared distribution with 1 degree of freedom. Hence, the $P_{\mathrm{FA}}$ is given by:

$$
\begin{aligned}
P_{\mathrm{FA}}\left[\mathcal{T}_{\mathrm{s}}^{\hat{\mathrm{x}}_{b}}\left(\mathbf{y}_{f}\right)\right] & =p\left(\mathcal{T}_{\mathrm{s}}^{\hat{\mathbf{x}}_{b}}\left(\mathbf{y}_{f}\right)>\xi_{\mathrm{s}} \mid \mathcal{H}_{0}\right) \\
& =1-p\left(2 \ln \left[\mathcal{T}_{\mathrm{s}}^{\hat{\mathbf{x}}_{b}}\left(\mathbf{y}_{f}\right)\right]<2 \ln \left(\xi_{\mathrm{s}}\right) \mid \mathcal{H}_{0}\right) \\
& =1-\Phi_{\chi_{1}^{2}}\left[2 \ln \left(\xi_{\mathrm{s}}\right)\right],
\end{aligned}
$$

where $\Phi_{\chi_{1}^{2}}$ denotes the Cumulative Density Function (CDF) of a $\chi^{2}{ }_{1}$ distribution. $\square$

We now aim at generalizing this result to the $\mathcal{T}_{\mathrm{cs}}^{\mathcal{B}}$ test. Let us recall that this test is the product of several $\mathcal{T}_{\mathrm{s}}^{\hat{\mathbf{x}}_{b}}$, meaning that their log will sum up.

Proposition 2.2. Given the reference set $\mathcal{B}$ and the corresponding $\left(\widehat{\mathbf{x}}_{b}\right)_{b \in \mathcal{B}}$, the compound GLR test with similarity constraint, noted $\mathcal{T}_{\mathrm{cs}}^{\mathcal{B}}$, has an upper-bounded false alarm rate: there is an upper bound for the false alarm rate, which only depends on the test threshold.

Proof. Assuming the $\mathbf{y}_{b}$ are independent leads, in a similar fashion than in Eqs. (23) and (25), to a $\chi^{2}$ dependence:

$P_{\mathrm{FA}}\left[\mathcal{T}_{\mathrm{cs}}^{\mathcal{B}}\left(\mathbf{y}_{f}\right)\right]=1-\Phi_{\chi_{|\mathcal{B}|}^{2}}\left[2 \ln \left(\xi_{\mathrm{cs}}\right)\right]$

where $|\mathcal{B}|$ stands for pixel number in the bright detection region $\mathcal{B}$, meaning that the $\chi^{2}$ distribution degrees of freedom is the number of bright spectra initially detected. However, assuming independence between the bright spectra is often unrealistic; in particular when including local spatial neighborhood (see Eq. (14)). Therefore, the degrees of freedom $k$ of the $\chi^{2}$ distribution is at most $|\mathcal{B}|$. Then Eq. (26) leads to the following bounding:

$P_{\mathrm{FA}}\left[\mathcal{T}_{\mathrm{cs}}^{\mathcal{B}}\left(\mathbf{y}_{f}\right)\right] \leq 1-\Phi_{\chi_{|\mathcal{B}|}^{2}}\left[2 \ln \left(\xi_{\mathrm{cs}}\right)\right]$

This means that for a given threshold $\xi_{\mathrm{cs}}$, a maximum type I error rate can be estimated. Numerical experiments will be presented in Section 3.3, supporting these analytical results.

Let us remark that the properties presented in this section hold when the references $\left(\widehat{\mathbf{x}}_{b}\right.$ or $\left.\left(\hat{\mathbf{x}}_{b}\right)_{b \in \mathcal{B}}\right)$ do not depend on the observations. The practical application presented in this paper requires to estimate the references from the observations, but other applications could benefit from, e.g., extra input data.

\section{Numerical results}

In this section, we quantify the method performance. Simulated HSI were developed for this purpose, and are described in Section 3.1. The method performance is then reported and analyzed in Section 3.2. The performance is compared with an ACE variant and with the SSGRF method from [19]. To our knowledge, there is no other method aiming at the detection of spatially wide sources in HSI. Finally, the tests distributions are estimated and discussed in Section 3.3.

\subsection{HSI simulation}

Using astronomical HSI implies that there is no available ground truth. The validation on real data can be carried out by experts; however this is difficult when the objects have not yet been observed. Hence, we have to build synthetic data in which the object of interest is known, to ensure a proper method validation. The simulated HSI, spatially consist of a bright elliptical (the galaxy) and a faint circular (the halo) emission, with intensities vanishing from the center of the object: a truncated Gaussian is used, setting the brightness to zero outside of the objects. The two elements have a similar spectral emission, the circular emission being fainter. The datacube $\left(\mathbf{y}_{s}\right)_{s \in \mathcal{S}}$ has $50 \times 50$ pixels and 50 spectral bands, and are centered on the objects. Fig. 1 provides insight of a simulated HSI.

A multivariate white Gaussian noise is then added. The SNR is defined as:

$\mathrm{SNR}=10 \log _{10}\left(\frac{\left\|\mathbf{x}_{\text {halo }}\right\|_{2}^{2}}{\operatorname{Tr}(\mathbf{\Sigma})}\right)$

where $\mathbf{x}_{\text {halo }}$ is the brightest underlying halo spectrum, and $\mathbf{\Sigma}$ is the noise 

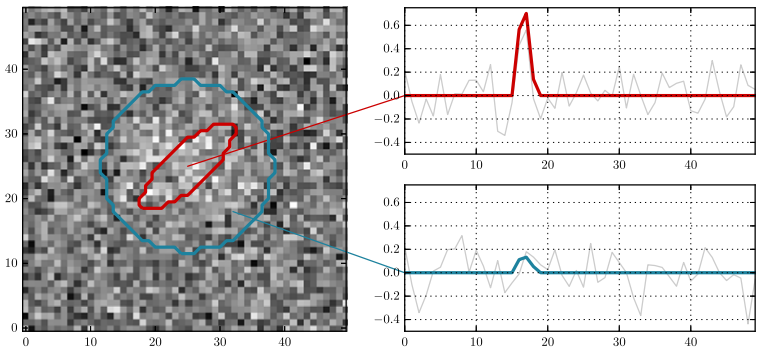

Fig. 1. Simulation example with $\mathrm{SNR}=-3 \mathrm{~dB}$. Left: HSI spectral average. The ellipse (resp. circle) represents the bright (resp. faint extended) elements. The corresponding spectra are represented in the right panel (dotted: noiseless, plain: observed).

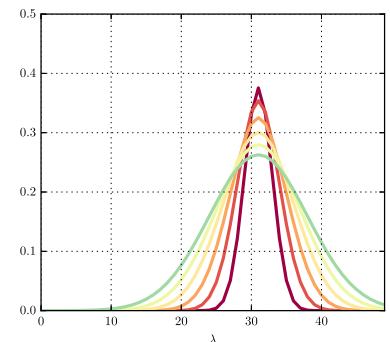

(a)
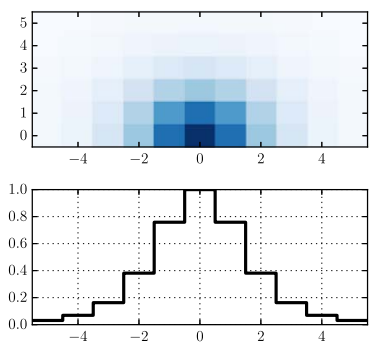

(b)
Fig. 2. Left: instances of six lines of the catalog at a given position. Right: half spatial FSF with its $1 \mathrm{D}$ middle profile.

covariance matrix. This definition is similar to a peak-SNR, meaning that this SNR is a maximum value with respect to other spectra.

As stated in Section 2, the catalog is a collection of normed Gaussian lines. The means range in the cube spectral dimension (150 ), and standard deviations range from 0.1 to 5 spectral bands (Fig. 2(a)). In the application context, thin emission lines are searched for. Whereas they may be asymmetric, they are unknown an may be undetectable at low SNR. Hence, Gaussian modeling is sufficient in this context.

The FSF is described by a 2D Moffat function [38], which is more heavy-tailed than a Gaussian and more accurate for modeling astrophysics instruments' FSF. It has two wavelength-dependent parameters, considered as constant in the simulated HSI: $a=2.0$ and $b=2.6$ (Fig. 2(b)).

\subsection{Performance evaluation}

The results presented in this section are estimated on 100 simulations, with different noise realizations, and the HSI are spectrally whitened before processing. This ensure that the spectral crosscorrelation are negligible, and allows us to consider the covariance matrix estimate diagonal. Besides, assuming the signals of interest are weak implies that errors in the covariance estimation prior to whitening are small. Furthermore, the FSF is assumed known, resulting e.g. from physical modeling. Note that the FSF is generally parametrically estimated: this implies that estimation errors are related to FSF parameters and not FSF coefficients, ensuring some properties (smoothness, fading to zero, peak at the center) are always verified. Therefore, the impact on the error is weak.

Bright Source Detection. The results from Fig. 3 bring a clear illustration that expanding a local neighborhood enhances the detection at low SNR: the difference between detection probabilities ranges from $23 \%(-20 \mathrm{~dB})$ to $75 \%(-10 \mathrm{~dB})$ of the recovered detection. Note that at high SNR, expanding the spatial neighborhood seems to limit
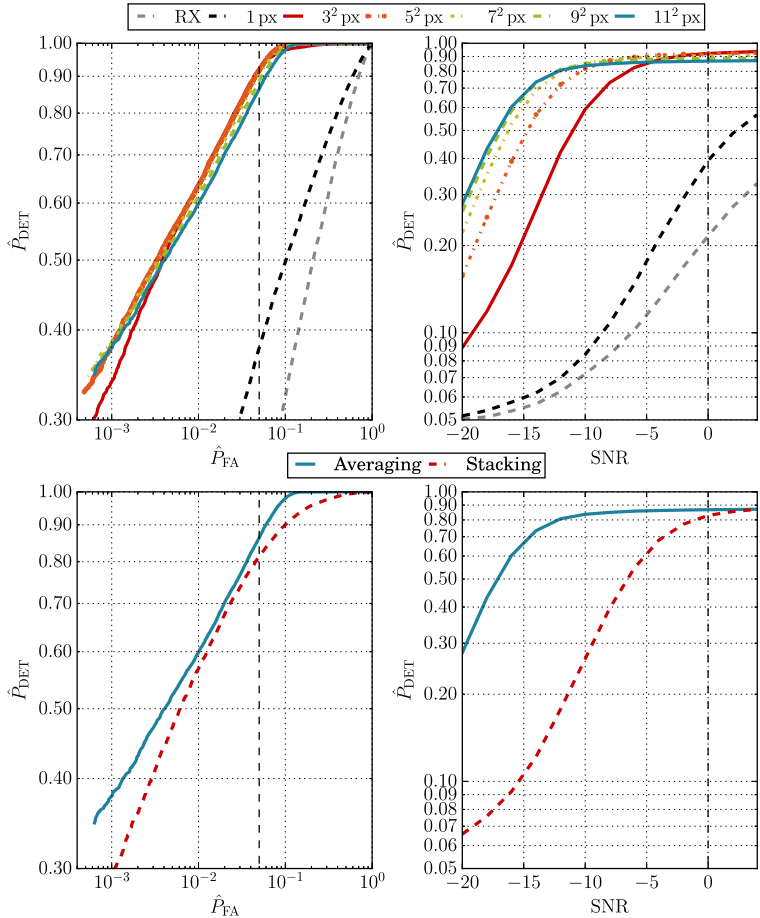

Fig. 3. Numerical results for the bright source detection. First column: ROC curves at SNR $=0 \mathrm{~dB}$; second column: $\widehat{P}_{\mathrm{DET}}$ with $\widehat{P}_{\mathrm{FA}}=0.05$. The first line investigates the use of spatial features, and the second line present estimations with multiple (50) observations, stacked or averaged, with a fixed window size of $11 \mathrm{px}$. Vertical dashed black line indicates the correspondences between ROC curves at $0 \mathrm{~dB}$ and $\widehat{P}_{\mathrm{FA}}=0.05$.

the detection power, because of increasing border effects. Note also that the RX algorithm [15] is outperformed even without considering the local neighborhood, likely because the anomaly in the simulated HSI has low energy.

We also observe in Fig. 3 that using multiple observations lowers method performance. Indeed, searching for the maximum in Eq. (17) heavily relies on the SNR, and with stacked multiple observations the SNR for a given spectrum is considerably lowered. The conclusion on this point is that, when using multiple observations, the max operator has to be avoided.

Compound Similarity Constrained GLR Test. The graphs from Fig. 4 illustrate the performance gain brought by the spatial features. One can in particular notice that 1) using only a 3-pixel-wide FSF window already greatly improves results; and 2) that due to FSF fading, the gain of using wider windows diminishes as the windows size increases.

The conclusion drawn from the bright source detection is applied when investigating the multiple observations features. Indeed, it relies in practice on the successive estimations of the coefficient $\alpha_{b}$ - based on the max operator - and the similarity coefficient $\beta_{f, b}$ (see Eq. (8)). We propose then to use the former on the averaged datacube and the latter on the stacked observations. Results are presented in Fig. 4. They show that using jointly multiple observations does not enhance the detection at a significant level. In this setting, using an observation-wise setting is counter-balanced by the loss induced by low observation-wise SNR.

Detection strategy. We now detail the global method for detection. As stated before, the two main steps consist in the bright source detection followed by the faint extended source detection. Note also that each step uses different whitened data: for the first step the complete datacube is whitened, as in the second the whitening addresses the spectra not already detected (in $\mathcal{F}$ ). This ensure that the covariance estimations are not contaminated by the bright spectra 

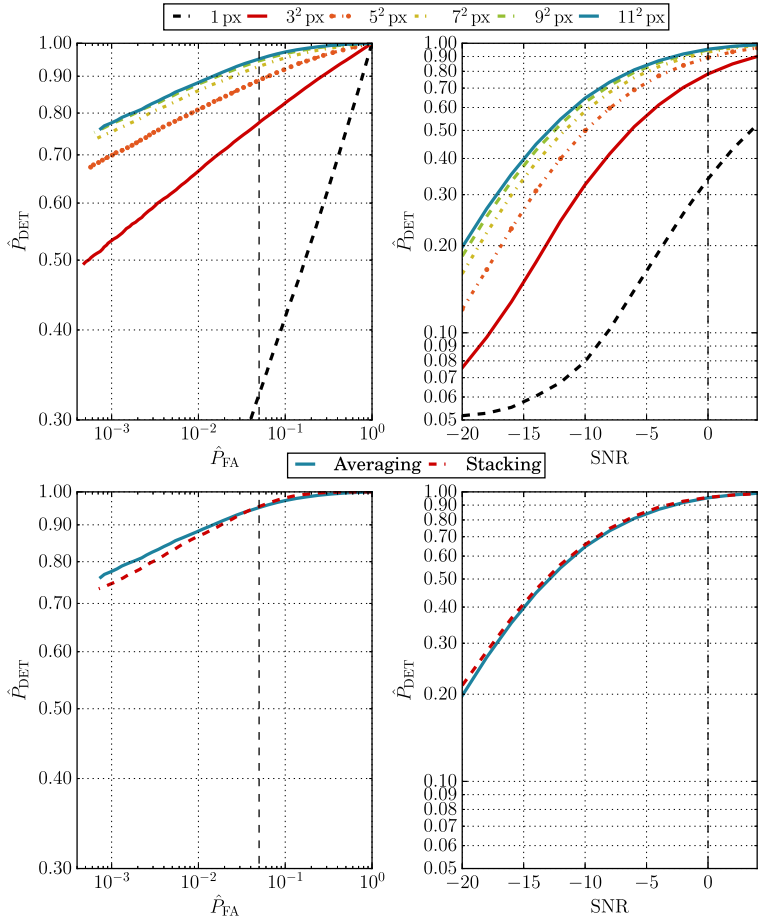

Fig. 4. Numerical results for extended faint source detection. The legend is the same as in Fig. 3.

set $\mathcal{B}$. Furthermore, detecting all bright pixels at the first step is not necessary, but it is desirable that there is no false detection to avoid further error propagation. To enforce this, a processing step is added, removing from $\mathcal{B}$ all spectra that are not spatially connex to the central source. This does not impede the detection strategy, as these spectra are recovered in the second step. The complete detection strategy is summarized in Algorithm 1.

\section{Algorithm 1. Full detection strategy.}

Input: Source cube $\mathbf{Y}$, target maximum $P_{\mathrm{FA}}$, catalog $\mathbf{C}, \mathrm{FSF}$ matrix $\mathbf{F}$.

Output: Detection map $C$.

1. Y spectral whitening on the $\mathcal{S}$ set.

2. Bright detection: $\mathcal{T}_{\mathrm{n}}\left(\mathbf{y}_{s}\right) \forall s \in \mathcal{S}$ (Eq. (17)), producing $\mathcal{B}$.

3. Preprocessing: remove pixels non-connex to the central one, producing $\mathcal{B}_{1}$.

4. Y spectral whitening on the $\mathcal{S} \backslash \mathcal{B}_{1}$ set.

5. Extended detection: $\mathcal{T}_{\text {ncs }}^{\mathcal{B}_{1}}\left(\mathbf{y}_{f}\right) \forall f \in \mathcal{S} \backslash \mathcal{B}_{1}$ (Eq. (18)), producing C.

Strategy performance is presented in Fig. 5, and its behavior is close to the results explained previously: considering spatial relationship gains up to $71 \%$ on detection probabilities, and considering multiple observations does not provide visible gain. At low SNR, we observe interesting results: $\widehat{P}_{\mathrm{DET}}=70 \%$ at $-10 \mathrm{~dB}, \widehat{P}_{\mathrm{DET}}=49 \%$ at $-15 \mathrm{~dB}$ when $\widehat{P}_{\mathrm{FA}}=5 \%$. One can also notice that the overall performance seems improved, as a result of using both halo and galaxy as the reference ground truth, instead of the halo without the galaxy. Finally, it should be noted that using multiple observations still does not provide noticeable gain.

We now address the comparison of the proposed detection strategy with other methods. First, results concerning a random field geometric modeling method [19] on the same simulated data are also presented. This method proposes a Spatio-Spectral Gaussian Random Field (SS-
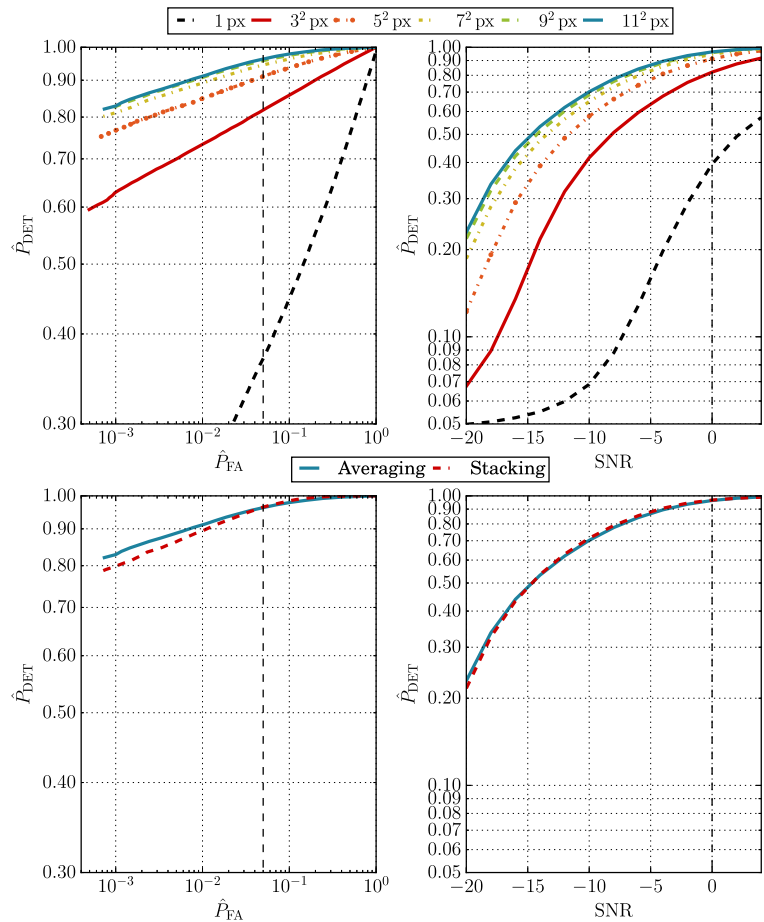

Fig. 5. Numerical results for the full strategy. Same legend as in Figs. 3 and 4 .

GRF) model for the background, accounting for the local spatial relationship between pixels.

Other well-known dectors in HSI are AMF [7] and ACE [8], both requiring the knowledge of a reference spectra. For comparison purpose, we design a second detector based on ACE. We use the detection strategy from Algorithm 1 and replace the last step (5. Extended detection) with an ACE detector, using as reference the averaged spectrum from the $\mathcal{B}_{1}$ region. Let us add that experiments with an AMF variant yields similar performance than the presented ACE variant.

Fig. 6 illustrates the comparison between the proposed method, its ACE variant and the SS-GRF model. Our method clearly outperforms SS-GRF modeling. This is explained by the fact that our application targets spectrally-constrained signals, accounts for wide spatial dependence, and is in this sense more specific than SS-GRF modeling. On the other hand, we observe that the ACE variant of the detection strategy offers slightly better results than our method at SNR $=0 \mathrm{~dB}$. However, this is not the case at low SNR, where there is noticeable gain between the proposed method and its ACE variant. This may be due to a

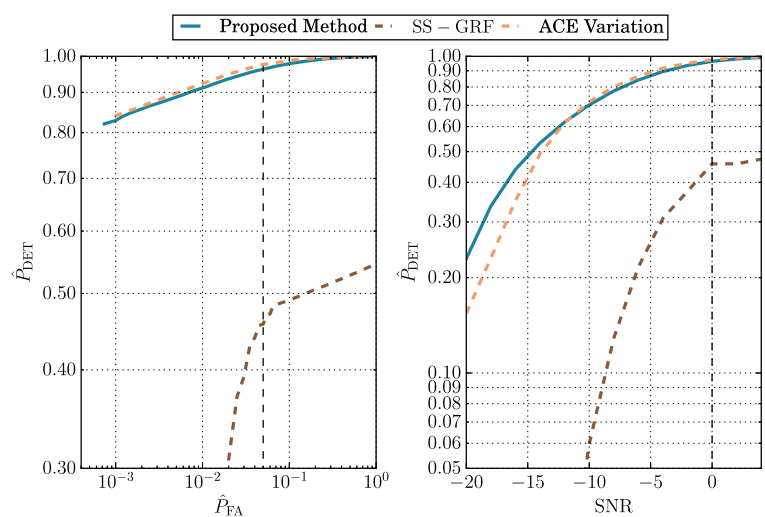

Fig. 6. Comparison of the proposed method with its ACE variant and with the SS-GRF method [19]. Same legend as in the first rows of Figs. 3, 4 and 5. 

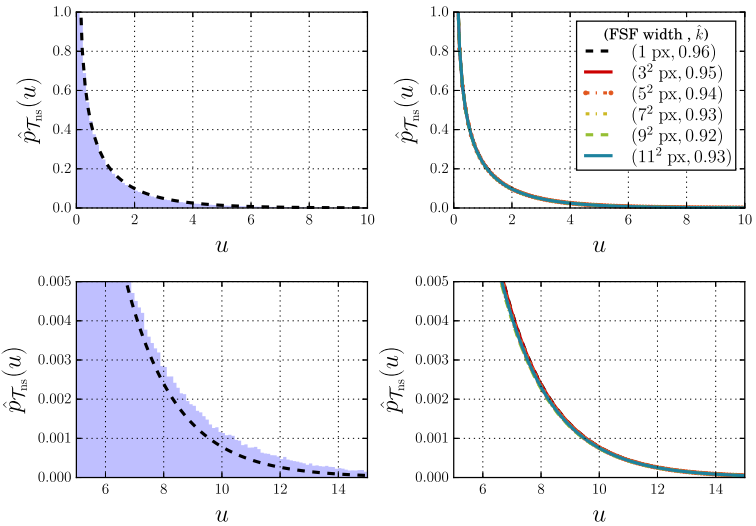

Fig. 7. Empirical $\mathcal{T}_{\mathrm{s}}^{\hat{\mathbf{x}}_{b}}$ and $\mathcal{T}_{\text {ns }}^{\hat{\mathbf{x}}_{b}}$ statistics under $\mathcal{H}_{0}$. The results were obtained with $10^{6}$ spectra realizations under white noise. Left: histograms (blue area) and ML estimation best fits of the tests without using spatial relationship. Right: ML estimation best fits of for varying spatial FSF windows. The second row focus on the distribution tails. Estimated degrees of freedom $\hat{k}$ for each fit are also given in the legend box. (For interpretation of the references to color in this figure legend, the reader is referred to the web version of this article.)

lowering of the ACE reference estimation quality when the SNR decreases.

To sum up, the main conclusions from the experiments are:

1. the use of spatial features greatly enhances the detection, up to a gain of $71 \%$ map proportion recovering;

2. using multiple observations is, in this framework, not an advantage because of the observation-wise lower SNR;

3. at very low SNR, the proposed strategy still provides good results.

We now detail experimental results concerning the link between the test thresholds and their false alarm rates.

\subsection{Tests statistics distributions}

This section reports numerical results on the test statistics $\mathcal{T}_{\mathrm{s}}^{\hat{\mathrm{x}}_{b}}$ and $\mathcal{T}_{\text {cs. }}^{\mathcal{B}}$. The main goal is to characterize their behavior under $\mathcal{H}_{0}$ with respect to the theoretical results expressed in Section 2.4. The tests are performed on simulated datacubes containing noise only, given a complete bright spectra set $\mathcal{B}$ (red ellipse in Fig. 1). A point of interest is the variation of the test statistics when the spatial neighborhood windowing varies.

Fig. 7 presents the results for the single $\mathcal{T}_{\mathrm{s}}^{\hat{\mathbf{x}}_{b}}$ test and its spatial
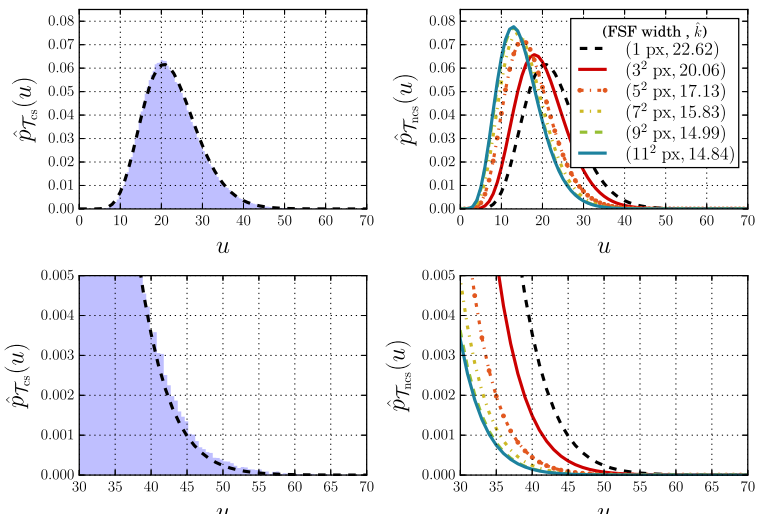

Fig. 8. Empirical $\mathcal{T}_{\mathrm{cs}}^{\mathcal{B}}$ and $\mathcal{T}_{\text {ncs }}^{\mathcal{B}}$ statistics under $\mathcal{H}_{0}$. The results were obtained with $10^{5}$ spectra realizations under white noise. Legend is the same as in Fig. 7. variations $\mathcal{T}_{\text {ns }}^{\hat{\mathbf{x}}_{b}}$. We first observe that, under $\mathcal{H}_{0}, \mathcal{T}_{\mathrm{s}}^{\hat{\mathbf{x}}_{b}}$ fits well its theoretical $\chi_{1}^{2}$ distribution. Let us notice that ML estimates slightly underestimates the distribution tails, with up to a $3 \cdot 10^{-4}$ gap. Furthermore, the distributions seem to be almost independent of the FSF window width, to the point of making ML estimation fits almost indistinguishable in Fig. 7.

In Fig. 8, the results for the compound $\mathcal{T}_{\mathrm{cs}}^{\mathcal{B}}$ and its spatial variations $\mathcal{T}_{\text {ncs }}^{\mathcal{B}}$ are presented. We also observe that the empirical data distribution fits well a chi-squared distribution. Besides, unlike the previous result, there is no visible gap between the estimated distribution and the histogram. Estimations of the ML distribution parameters reflect that as the FSF window grows, the degrees of freedom decrease. Indeed, a wider window implies that to describe the set $\mathcal{B}$, less spectra are required.

The results confirmed the properties expressed in Section 2.4 and in practice allow us to provide a maximum false alarm probability for the detection of astronomical sources. Results on real MUSE images, for which no ground truth is available, are presented in the next section.

\section{Application on real MUSE data}

In this section, we apply the method to MUSE observations. The MUSE instrument is set since 2014 at the ESO/VLT facility (Paranal, Chile). It provides observations with a spatial size of $300 \times 300 \mathrm{px}$ and $(0.2 \operatorname{arcsec})^{2}$ resolution, with 3640 spectral bands at each pixel and $1.25 \AA$ individual bandwidth. The total bandwidth covers $4750-9300 \AA$ and therefore makes it possible to see very distant, faint and small astronomical objects.

Due to its complex structure, the instrument was the object of several preliminary studies, including the noise behavior [39] and the instrument's Point Spread Function [40]. The main points are:

1. Due to an over long exposure time ( $1 \mathrm{~h}$ and more), the noise can be considered as non-correlated and Gaussian. Note that these assumptions are valid for the merged observation, but they are not for single observations, in which we observed that the noise is non-Gaussian and correlated.

2. The PSF is separable. The FSF is modeled as a Moffat, and is estimated from the observation of a bright star [36]. The LSF in the observations spreads over $2.1 \pm 0.2$ spectral bands.

The astronomical observation is available from [36]. The sources are manually located by experts (catalog available from [41]), and a total of $90 \mathrm{LAE}$ were found. Note that the data is very noisy and that extended sources (CGM) are visible only when merging narrow-band information [1].

For each source, an HSI spatially and spectrally centered on the LAE is extracted. Each HSI is processed independantly. To avoid contamination by a smooth or energetic component from neighbor object such as bright galaxy or star, spectrum-wise median subtractions are performed over \pm 150 bandwidths. This step satisfies the assumption of zero-mean noise. Then Algorithm 1 is applied on each HSI.

Fig. 9 presents results on six objects with the target $P_{\mathrm{FA}}$ level of $10^{-4}$. These figures illustrate the diversity of cases arising with real data:

1. Spatially, there is no common shape between detection maps, which may be narrow (ID 552) or wide (IDs 43, 92, 139), isotropic (IDs 43, 144) or not (IDs 92, 200) while being split in multiple blobs (IDs 139,552 ) or not (IDs 43, 92, 144, 200).

2. Spectrally, the averaged spectra show that while they all have an asymmetric emission line (the Lyman-alpha line), they differ in shape from one object to another. Since the spectra are averaged over the detection region, we observe that the SNR range strongly varies, from bright objects (ID 43, 144) to very faint signals (ID 200, $552)$. 

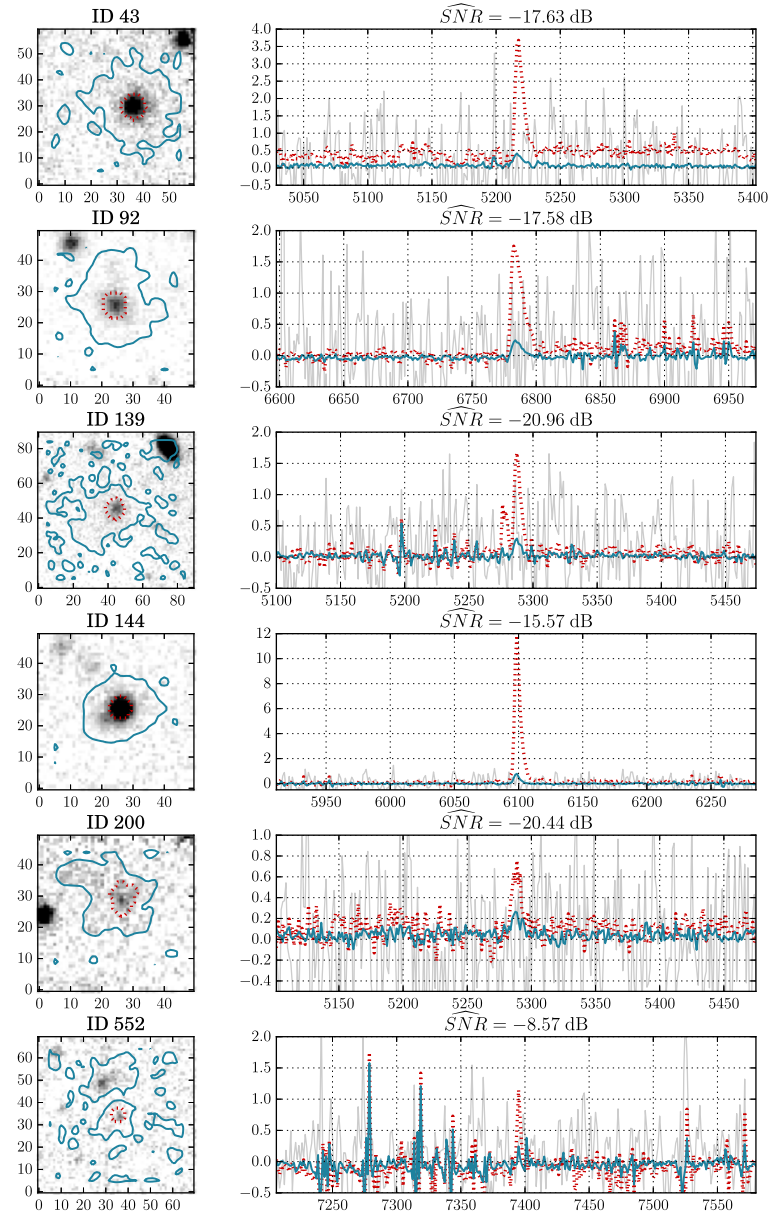

Fig. 9. Results on 6 real MUSE observations from HDFS [36] observation. Left: narrowband images (averaged datacube over 10 spectral bands). The dotted red contour is the bright source detection map. The solid blue contour is the strategy detection map, with target maximum $P_{\mathrm{FA}}$ at $10^{-4}$. Right: spectra averaged over the bright detection map (dotted red) and the proposed strategy detection map (solid blue). An outer spectrum (bottom right corner in the images) is shown in gray for comparison. Wavelengths are in Angström and intensities are in $10^{-20} \mathrm{ergs}^{-1} \mathrm{~cm}^{-2} \AA^{-1}$. Identification numbers are from the catalog available in [41]. (For interpretation of the references to color in this figure legend, the reader is referred to the web version of this article.)

These results highlight the challenges faced by CGM study. They show that using priors on CGM shape is unrealistic, while spectral behavior can subtly vary from one object to another, preventing a fine general emission line modeling. Despite these challenges, the method appears to be consistent: we observed that varying the target $P_{\mathrm{FA}}$ from $10^{-2}$ to $10^{-4}$ induces only minor changes (few pixels) to the detection maps. This means that the detection strategy provides a good contrast between signals and background, which is the goal of the proposed method.

\section{Conclusion}

This paper has presented a two-step method for faint extended source detection in HSI, with application to CGM detection in astronomical data from the MUSE instrument. The methods behavior and theoretical properties were detailed, providing maximum type I error probability for the second step of the detection strategy. Results on simulated data have allowed a proper method validation without hypothesis on the source morphology, and results on real MUSE data seem convincing. In the spirit of research reproducibility, the source code of our experiments will be made available.

\section{Acknowledgements}

J.-B. Courbot acknowledges support from the ERC advanced grant 339659-MUSICOS. This work was funded in part by the DSIM project under grant ANR-14-CE27-0005. The authors thank Roland Bacon (France, CNRS-CRAL) for providing the MUSE data and for fruitful discussions. They also thank Raphaël Bacher (France, CNRS-GIPSAlab) for interactions on the topic of CGM detection. They finally would like to thank Ola Ahmad (Canada, LIV4D) for providing SS-GRF source code and discussions.

\section{References}

[1] L. Wisotzki, R. Bacon, J. Blaizot, J. Brinchmann, E.C. Herenz, J. Schaye, N. Bouché, S. Cantalupo, T. Contini, C.M. Carollo, J. Caruana, J.-B. Courbot, E. Emsellem, S. Kamann, J. Kerutt, F. Leclercq, S.J. Lilly, V. Patrício, C. Sandin, M. Steinmetz, L.A. Straka, T. Urrutia, A. Verhamme, P.M. Weilbacher, M. Wendt, Extended lyman $\alpha$ haloes around individual high-redshift galaxies revealed by MUSE, Astron. Astrophys. 587 (2016) A98.

[2] S. Bourguignon, D. Mary, É. Slezak, Restoration of astrophysical spectra with sparsity constraints: models and algorithms, IEEE J. Sel. Top. Signal Process. 5 (5) (2011) 1002-1013.

[3] S. Park, N. Dobigeon, A.O. Hero, Variational semi-blind sparse deconvolution with orthogonal kernel bases and its application to MRFM, Signal Process. 94 (2014) 386-400.

[4] Y. Benjamini, Y. Hochberg, Controlling the false discovery rate a practical and powerful approach to multiple testing, J. R. Stat. Soc. Ser. B (Methodol.) (1995) 289-300.

[5] C. Meillier, F. Chatelain, O. Michel, H. Ayasso, Error control for the detection of rare and weak signatures in massive data, in: Signal Processing Conference (EUSIPCO), 2015 23rd European, IEEE, 2015, pp. 1974-1978.

[6] J.B. Broadwater, R. Chellappa, Adaptive threshold estimation via extreme value theory, IEEE Trans. Signal Process. 58 (2) (2010) 490-500.

[7] I. Reed, J. Mallett, L. Brennan, Rapid convergence rate in adaptive arrays, IEEE Trans. Aerosp. Electron. Syst. 6 (1974) 853-863.

[8] S. Kraut, L.L. Scharf, L.T. McWhorter, Adaptive subspace detectors, IEEE Trans. Signal Process. 49 (1) (2001) 1-16.

[9] E. Truslow, D. Manolakis, M. Pieper, T. Cooley, M. Brueggeman, Performance prediction of matched filter and adaptive cosine estimator hyperspectral target detectors, IEEE J. Sel. Top. Appl. Earth Obs. Remote Sens. 7 (6) (2014) 2337-2350.

[10] S.M. Kay, Fundamentals of statistical signal processing: Detection theory, vol. 2, Prentice-Hall PTR, 1998.

[11] C.-I. Chang, Orthogonal subspace projection (OSP) revisited: a comprehensive study and analysis, IEEE Trans. Geosci. Remote Sens. 43 (3) (2005) 502-518.

[12] B. Du, Y. Zhang, L. Zhang, D. Tao, Beyond the sparsity-based target detector: a hybrid sparsity and statistics-based detector for hyperspectral images, IEEE Trans. Image Process. 25 (11) (2016) 5345.

[13] L. Zhang, L. Zhang, D. Tao, X. Huang, B. Du, Hyperspectral remote sensing image subpixel target detection based on supervised metric learning, IEEE Trans. Geosci. Remote Sens. 52 (8) (2014) 4955-4965.

[14] L. Zhang, L. Zhang, D. Tao, X. Huang, Sparse transfer manifold embedding for hyperspectral target detection, IEEE Trans. Geosci. Remote Sens. 52 (2) (2014) 1030-1043.

[15] I. Reed, Y. Xiaoli, Adaptive multiple-band CFAR detection of an optical pattern with unknown spectral distribution, IEEE Trans. Acoust. Speech Signal Process. 38 (10) (1990) 1760-1770.

[16] H. Kwon, N.M. Nasrabadi, Kernel RX-algorithm: a nonlinear anomaly detector for hyperspectral imagery, IEEE Trans. Geosci. Remote Sens. 43 (2) (2005) 388-397.

[17] J.A. Malpica, J.G. Rejas, M.C. Alonso, A projection pursuit algorithm for anomaly detection in hyperspectral imagery, Pattern Recognit. 41 (11) (2008) 3313-3327.

[18] A. Huck, M. Guillaume, Asymptotically CFAR-unsupervised target detection and discrimination in hyperspectral images with anomalous-component pursuit, IEEE Trans. Geosci. Remote Sens. 48 (11) (2010) 3980-3991.

[19] O. Ahmad, C. Collet, F. Salzenstein, Spatio-spectral gaussian random field modeling approach for target detection on hyperspectral data obtained in very low SNR, in: Image Processing (ICIP), 2015 IEEE International Conference on, IEEE, 2015.

[20] W. Sakla, A. Chan, J. Ji, A. Sakla, An svdd-based algorithm for target detection in hyperspectral imagery, IEEE Geosci. Remote Sens. Lett. 8 (2) (2011) 384-388.

[21] B. Du, L. Zhang, Target detection based on a dynamic subspace, Pattern Recognit. 47 (1) (2014) 344-358.

[22] Y. Xu, Z. Wu, J. Li, A. Plaza, Z. Wei, Anomaly detection in hyperspectral images based on low-rank and sparse representation, IEEE Trans. Geosci. Remote Sens. 54 (4) (2016) 1990-2000.

[23] E.J. Kelly, An adaptive detection algorithm, IEEE Trans. Aerosp. Electron. Syst. 2 (1986) 115-127.

[24] J. Neyman, E. Pearson, On the problem of the most efficient tests of statistical hypotheses, Philosophical Transactions of the Royal Society of London. Series A, Containing Papers of a Mathematical or Physical Character 231 (1933) pp. 289337.

[25] J. Fan, C. Zhang, J. Zhang, Generalized likelihood ratio statistics and Wilks phenomenon, Ann. Stat. 29 (1) (2001) 153-193. 
[26] S. Paris, D. Mary, A. Ferrari, PDR and LRMAP detection tests applied to massive hyperspectral data, in: Computational Advances in Multi-Sensor Adaptive Processing (CAMSAP), 2011 Proceedings of the 4th IEEE International Workshop on, IEEE, 2011, pp. 93-96.

[27] S. Paris, D. Mary, A. Ferrari, Detection tests using sparse models, with application to hyperspectral data, IEEE Trans. Signal Process. 61 (6) (2013) 1481-1494.

[28] S. Paris, R.F.R. Suleiman, D. Mary, A. Ferrari, Constrained likelihood ratios for detecting sparse signals in highly noisy 3d data, in: 2013 IEEE International Conference on Acoustics, Speech and Signal Processing, IEEE, 2013, pp. 39473951.

[29] B. Du, R. Zhao, L. Zhang, L. Zhang, A spectral-spatial based local summation anomaly detection method for hyperspectral images, Signal Process. 124 (2016) $115-131$.

[30] S. Schweizer, J. Moura, Efficient detection in hyperspectral imagery, IEEE Trans. Image Process. 10 (4) (2001) 584-597.

[31] C.C. Funk, J. Theiler, D. Roberts, C.C. Borel, et al., Clustering to improve matched filter detection of weak gas plumes in hyperspectral thermal imagery, IEEE Trans. Geosci. Remote Sens. 39 (7) (2001) 1410-1420.

[32] C. Meillier, F. Chatelain, O. Michel, H. Ayasso, Nonparametric Bayesian extraction of object configurations in massive data, IEEE Trans. Signal Process. 63 (8) (2015) 1911-1924.

[33] C. Meillier, F. Chatelain, O. Michel, R. Bacon, L. Piqueras, R. Bacher, H. Ayasso, Selfi: an object-based, bayesian method for faint emission line source detection in muse deep field data cubes, Astron. Astrophys.-AA 588 (2016) A140.
[34] R. Bacon, M. Accardo, L. Adjali, H. Anwand, S. Bauer, I. Biswas, J. Blaizot, D. Boudon, S. Brau-Nogue, J. Brinchmann, et al., The MUSE second-generation VLT instrument, in: SPIE Astronomical Telescopes+ Instrumentation, SPIE, 2010.

[35] J.-B. Courbot, V. Mazet, E. Monfrini, C. Collet, Detection of faint extended sources in hyperspectral data and application to HDF-S MUSE observations, in: 2016 IEEE International Conference on Acoustics, Speech and Signal Processing (ICASSP), IEEE, 2016, pp. 1891-1895.

[36] R. Bacon, J. Brinchmann, J. Richard, T. Contini, A. Drake, M. Franx, S. Tacchella, J. Vernet, L. Wisotzki, J. Blaizot, et al., The MUSE 3D view of the hubble deep field south, Astron. Astrophys. 575 (2015) A75.

[37] M. Petremand, A. Jalobeanu, C. Collet, Optimal Bayesian fusion of large hyperspectral astronomical observations, Stat. Methodol. 9 (1) (2012) 44-54.

[38] A. Moffat, A theoretical investigation of focal stellar images in the photographic emulsion and application to photographic photometry, Astron. Astrophys. 3 (1969) 455.

[39] P. Weilbacher, O. Streicher, T. Urrutia, A. Jarno, A.Pécontal-Rousset, R. Bacon, P. Böhm, Design and capabilities of the MUSE data reduction software and pipeline, in: SPIE Astronomical Telescopes+ Instrumentation, SPIE, 2012.

[40] D. Serre, E. Villeneuve, H. Carfantan, L. Jolissaint, V. Mazet, S. Bourguignon, A. Jarno, Modeling the spatial PSF at the VLT focal plane for MUSE WFM data analysis purpose, in: SPIE Astronomical Telescopes+ Instrumentation, SPIE, 2010

[41] MUSE science website, 〈http://www.muse-vlt.eu/science〉, (accessed 18-01.17), 2017. 